\title{
Interplay between Laminar Specificity and Activity- Dependent Mechanisms of Thalamocortical Axon Branching
}

\author{
Naofumi Uesaka, ${ }^{*}$ Yasufumi Hayano, ${ }^{*}$ Akito Yamada, and Nobuhiko Yamamoto \\ Neuroscience Laboratories, Graduate School of Frontier Biosciences, Osaka University, Suita, Osaka 565-0871, Japan
}

\begin{abstract}
Target and activity-dependent mechanisms of axonal branching were studied in the thalamocortical (TC) projection using organotypic cocultures of the thalamus and cortex. TC axons were labeled with enhanced yellow fluorescent protein (EYFP) by a single-cell electroporation method and observed over time by confocal microscopy. Changes in the firing activity of cocultures grown on multielectrode dishes were also monitored over time. EYFP-labeled TC axons exhibited more branch formation in and around layer 4 of the cortical explant during the second week in vitro, when spontaneous firing activity increased in both thalamic and cortical cells. Time-lapse imaging further demonstrated that branching patterns were generated dynamically by addition and elimination with a bias toward branch accumulation in the target layer. To examine the relationship between neural activity and TC branch formation, the dynamics of axonal branching was analyzed under various pharmacological treatments. Chronic blockade of firing or synaptic activity reduced the remodeling process, in particular, branch addition in the target layer. However, extension of branches was not affected by this treatment. Together, these findings suggest that neural activity can modify the molecular mechanisms that regulate lamina-specific TC axon branching.
\end{abstract}

Key words: activity; axon guidance; cerebral cortex; lamina; thalamus; tissue culture

\section{Introduction}

During development, fundamental neural connections are established by developmentally regulated molecules that are expressed in particular spatiotemporal patterns, whereas neural activity modifies the basic connection patterns (Katz and Shatz, 1996; Cline, 2003). Thalamocortical (TC) axon branching in the mammalian neocortex is a well-characterized system in which to investigate these two mechanisms. To date, in vivo and in vitro studies demonstrated the existence of an axonal targeting mechanism for lamina-specific branch formation (Lund et al., 1977; Yamamoto et al., 1989; Molnár and Blakemore, 1991; Bolz et al., 1992; Ghosh and Shatz, 1992; Yamamoto et al., 1992, 1997; Agmon et al., 1993; Kageyama and Robertson, 1993; Catalano et al., 1996; Molnár and Blakemore, 1999). We further demonstrated that a membrane-associated molecule that is expressed in the target layer promotes branch formation, whereas a negative regulator is present across all cortical layers (Yamamoto et al., 2000). Branch formation of TC axons is also influenced by neural activity. The best characterized example is the effect of monocular deprivation, which induces the shrinkage of TC axon arbors serv-

Received 0ct. 27, 2006; revised April 9, 2007; accepted April 11, 2007.

This work was supported by Grants-in-Aid for Scientific Research Projects 15300107, 15029233, 17023030, and 18021021 from Japanese Ministry of Education, Culture, Science, and Sports and by Human Frontier Science Program Grant RGP0107/2001. We thank Dr. Edward S. Ruthazer for critical reading of this manuscript.

${ }^{*}$ N.U. and Y.H. contributed equally to this work.

Correspondence should be addressed to Nobuhiko Yamamoto, Neuroscience Laboratories, Graduate School of Frontier Biosciences, Osaka University, Suita, 0saka 565-0871, Japan. E-mail: nobuhiko@fbs.osaka-u.ac.jp.

N. Uesaka's present address: Department of Cellular Neuroscience, Graduate School of Medicine, Osaka University, Suita, Osaka 565-0871, Japan.

DOI:10.1523/JNEUROSCI.4685-06.2007

Copyright $\odot 2007$ Society for Neuroscience $\quad$ 0270-6474/07/275215-09\$15.00/0 ing the deprived eye and the expansion of nondeprived eye arbors (Hubel et al., 1977; Antonini and Stryker, 1996; Antonini et al., 1999). Although ocular dominance plasticity alters the tangential extent of TC axons without affecting layer specificity, it has been reported that a complete blockade of firing activity prevents even lamina-specific branching (Herrmann and Shatz, 1995). This suggests that both lamina-specific molecular mechanisms and activity-dependent mechanisms play a role in TC axon branching. However, how these two mechanisms cooperate with each other is essentially unknown.

To address this issue, we studied the activity dependency and laminar specificity of TC axon branching in organotypic cocultures of the thalamus and cortex. First, thalamic axon morphology was examined by imaging enhanced yellow fluorescent protein (EYFP)-labeled axons. Simultaneously, electrical activity of cortical and thalamic cells was monitored through the implanted electrodes of multielectrode dishes (MEDs). Second, the dynamics of axon branching was analyzed by time-lapse imaging to reveal positive and negative regulators of growth behavior. Finally, the influence of neural activity on the branching behavior was examined by pharmacological manipulation of firing and glutamatergic transmission. Our results suggest that TC axon branching is generated dynamically by addition and elimination, with a preference toward branch accumulation in the target layer, and that synaptic transmission is required for the remodeling process, in particular, branch addition in the target layer.

\section{Materials and Methods}

Organotypic slice culture. Thirty-two pregnant rats and 77 pups were used to prepare cocultures. All experiments were performed according to the guidelines laid down by the animal welfare committees of Osaka Univer- 
sity and the Japan Neuroscience Society. Cocultures of the cortex with the thalamic explants were prepared as described previously (Yamamoto et al., 1989, 1992). In brief, the dorsal thalamic region was dissected from embryonic day 15 rat embryos (Sprague Dawley; Clea Japan, Tokyo, Japan), and cortical slices were dissected from the visual and somatosensory cortices of postnatal day 1 or 2 rat. A block of the thalamus was plated at the ventricular side of the cortical slice on a membrane filter (Millicell-CM PICMORG50; Millipore, Bedford, MA), which was coated with rat tail collagen (Uesaka et al., 2005). To monitor neural activity, cortical and thalamic explants were plated on MEDs (Alpha MED Sciences, Tokyo, Japan) (Uesaka et al., 2005). The culture medium consisted of a 1:1 mixture of DMEM and Ham's F-12 (Invitrogen, Carlsbad, CA) with several supplements containing insulin and transferrin (Yamamoto et al., 1992). These cultures were maintained at $37^{\circ} \mathrm{C}$ in an environment of humidified $95 \%$ air and $5 \% \mathrm{CO}_{2}$.

Axon labeling by electroporation. To label individual thalamic axons in the cortical explants, a plasmid containing the coding region of EYFP (pCAGGS; a generous gift from Dr. Hatanaka, Nara Institute of Science and Technology, Nara, Japan) (Niwa et al., 1991) was transfected
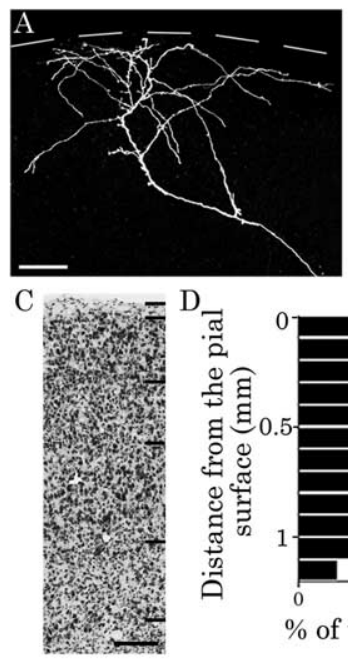

in each bin

$\mathrm{B}$ สี

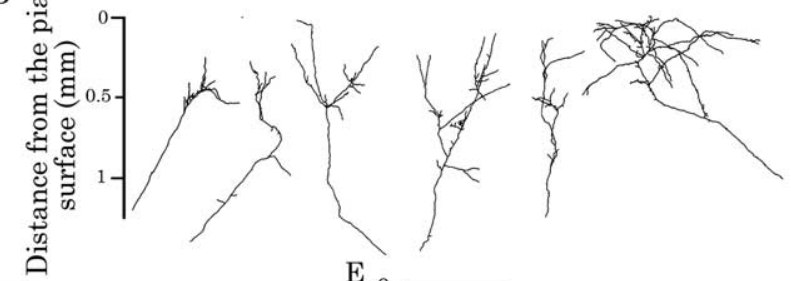

Figure 1. Thalamic axon branching in cocultures of the cortex with the thalamus. $A$, Branching of an EYFP-labeled TC axon. Scale bar, $200 \mu \mathrm{m}$. B, Typical examples of individual TC axons after 2 weeks in vitro are shown. $\boldsymbol{C}$, Nissl-stained section of the cortical explant. Lines indicate the borders of layers $1,2 / 3,4,5$, and 6 . Scale bar, $200 \mu \mathrm{m}$. D, Laminar distribution of branch points as a function of the depth in the cortex ( 36 cultures, 45 axons). Distribution histogram of branch points is constructed from $100 \mu \mathrm{m}$ bins. Arrows indicate the average the layer 4 borders. $E$, Laminar distribution of the total length of branches contained in each bin is indicated as a function of the depth in the cortex ( 36 cultures, 45 axons). Interrupted lines represent the minimal length (100 $\mu \mathrm{m})$ in each bin. Error bars show SEM. ${ }^{*} p<0.05$ and ${ }^{* *} p<0.01$, significant differences from the branch length in the cortical depth of $700-800 \mu \mathrm{m}$. into a small number of thalamic cells using the

method described previously (Uesaka et al., 2005). This method made it possible to observe individual axonal morphologies for $>1$ week. In brief, the plasmid solution $(1-2 \mu \mathrm{g} / \mu \mathrm{l})$ was applied to the thalamic explants through a glass micropipette (tip diameter of $50 \mu \mathrm{m}$ ), and then electrical pulses (five to seven trains of 200 square pulses of $1 \mathrm{~ms}$ duration at $200 \mathrm{~Hz}, 500-700 \mu \mathrm{A}$ ) were delivered across a silver wire placed inside a glass micropipette (tip diameter of $200-300 \mu \mathrm{m}$ ) and a silver wire ground electrode placed in the culture medium. The electroporation was applied to two or three sites of each thalamic explant.

EYFP-labeled axons were observed using an upright laser scanning confocal microscope (MRC-600; Bio-Rad, Tokyo, Japan) equipped with air objective lenses ( $10 \times$ and $40 \times$; working distance of 5-6 mm) and a filter set for fluorescein isothiocyanate. EYFP fluorescence remained high for $>1$ week, even with repeated imaging. A $z$-series of 2-20 optical sections were sampled (1-5 $\mu \mathrm{m}$ interval). At the end of the experiment, images were taken at lower magnification ( $4 \times$ objective) to determine the locations of axons in the cortical explants.

Quantification of axon branching. Individually distinguishable axons that reached or passed through layer 4 were selected and drawn using NIH Image. All measurements of axonal morphology such as branch length were performed using custom NIH Image software macros. Small processes $(<5 \mu \mathrm{m})$ from the axon shaft were excluded from the analysis. The following parameters were measured: the number of branch points, the lengths of individual branch tip segments measured from the branch tips to their nearest branch points, and branch length in each $0.1 \mathrm{~mm}$ depth (Uesaka et al., 2005). The laminar locations of branch points were determined based on lower-magnification pictures and Nissl-stained sections. Then, the cortical thickness was divided in $100 \mu \mathrm{m}$ bins, and the number of branch points was counted in each bin. The total length of axonal processes contained in each bin was also measured.

Time-lapse study. To examine branch dynamics of growth or retraction, EYFP-labeled axons were observed every $24 \mathrm{~h}$ by confocal microscopy. The observation was performed for $\sim 5 \mathrm{~min}$ at room temperature, and cultures were put back in the incubator.

The obtained images by time-lapse study were analyzed by NIH images as described above. The branches that grew or retracted $<5 \mu \mathrm{m}$ over $24 \mathrm{~h}$ were classified as stable branches. Branches that were present on both one day and the next day but became longer were termed as elongating branches. Branches that did not exist on one day but were newly added until the next day were termed as added branches. Branches that were seen on both one day and the next day but became shorter by the next day were termed as retracting branches. Branches that were detected on one day and disappeared completely by the next day were termed as lost branches.

Histology. Laminar configuration in coculture preparations was determined by Nissl staining (Yamamoto et al., 1989, 1992). The culture slices were fixed by immersion in $4 \%$ paraformaldehyde in $0.1 \mathrm{~m}$ phosphate buffer and kept in $30 \%$ sucrose in PBS. The cultures were embedded on an agar block (4\% agar in PBS), cut into $20 \mu \mathrm{m}$ frozen sections, and stained with cresyl violet.

Recording and quantitative analysis of spontaneous activity. To examine spontaneous activities of cortical and thalamic cells, extracellular recording was performed on MEDs from several locations simultaneously as described previously (Shimono et al., 2002; Uesaka et al., 2005). In brief, the extracellular voltages at the electrodes were amplified and stored in a hard disk after digitization. The frequency of spontaneous firing activity was quantified with Axograph software (Molecular Devices, Palo Alto, CA). Negative potentials with amplitudes above a set threshold of 1.5 times the maximal amplitude of the baseline noise $(20 \mu \mathrm{V})$ were counted as one spike. To analyze the development of the firing activity quantitatively, four to six electrodes per one coculture preparation were used.

Pharmacological treatments. To manipulate firing and synaptic activity, culture media containing the following drugs were applied between $7 \mathrm{~d}$ in vitro (DIV) and 15 DIV: D-APV $(100 \mu \mathrm{M})$, DNQX (20 $\mu \mathrm{M}$; Tocris Cookson, Bristol, UK), and TTX (100 nm; Seikagaku-Kogyo, Toyko, Japan). Drug-containing media was exchanged every other day.

Statistical analysis. All statistical values obtained from the morphological and electrophysiological experiments were presented as mean \pm SEM. For all features measured, evaluation of the differences among groups has been obtained by comparing groups two at a time, using the Mann-Whitney $U$ test for nonparametric statistical analysis.

\section{Results}

Branching pattern of TC axons in the cultured cortex

TC axon branching in cocultures of the thalamus with cortex was examined by introducing EYFP-encoding plasmid into a small number of thalamic cells. As shown in Figure 1, $A$ and $B$, most of the individually distinguishable EYFP-labeled axons formed 

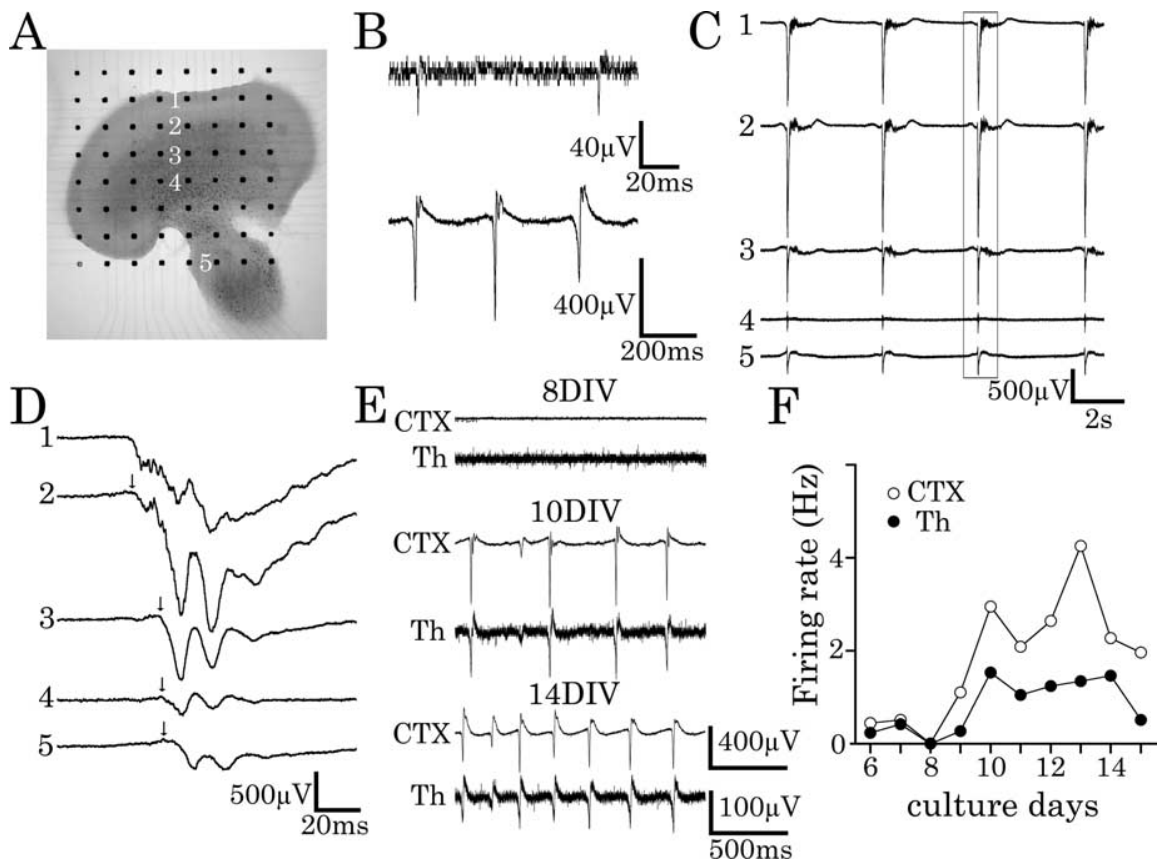

Figure 2. Spontaneous activity in cocultures. $\boldsymbol{A}$, The coculture of the cortex with the thalamus positioned on the MED. The thalamic explant is placed at the ventral side of the cortex. Microelectrodes are arranged in an $8 \times 8$ array with an interpolar distance of $300 \mu \mathrm{m}$. B. Single-unit (top trace) and field potential activity (bottom trace) recorded within cortical slices. $\boldsymbol{C}$, Spontaneous activity recorded from five electrodes within cortical (top 4 traces) and thalamic (bottom trace) explants. The trace number corresponds to those shown in $\boldsymbol{A}$. D, The traces framed by the rectangle in $\boldsymbol{C}$ are expanded. Arrows represent the onset of activity at each electrode. $\boldsymbol{E}$, Activity patterns recorded from the same slice after 8, 10, and 14 DIV are shown. Activity of cortical (CTX; top traces) and thalamic (Th; bottom traces) cells increases as development progresses. $F$, Mean firing rates of spontaneous activity in cortical and thalamic explants are shown as a function of culture days ( 5 cultures). Open and filled circles represent the firing rates of cortical and thalamic cells, respectively.

elaborate branches (branch points per axon, $16.7 \pm 2.2 ; n=45$ ) in cortical explants after 2 weeks in vitro. A quantitative analysis demonstrated that branch points were distributed with a peak at $300-500 \mu \mathrm{m}$ from the pial surface (Fig. $1 C, D$ ), corresponding to cortical layer 4 (Yamamoto et al., 1992). The branch length in each $0.1 \mathrm{~mm}$ bin (see Materials and Methods) was also greater in the similar laminar locations, although the distribution was slightly broader (Fig. 1E). These observations indicate that TC axon branches are formed in vitro with laminar specificity resembling that found in vivo (Ghosh and Shatz, 1992; Agmon et al., 1993; Kageyama and Robertson, 1993; Catalano et al., 1996), in accordance with previous findings (Yamamoto et al., 1989, 1992; Molnár and Blakemore, 1991; Bolz et al., 1992).

TC axon branching was also studied after just 1 week in vitro. The majority of labeled axons reached the upper layers but had as yet undergone little branching (supplemental Fig. 1, available at www.jneurosci.org as supplemental material). Only half of the TC axons had one or more branches. The mean number of branch points per axon was $1.2 \pm 0.4(n=12)$. Therefore, the majority of TC axonal branches appear to emerge during the second week in vitro, which is consistent with the finding that branch formation was increased with the age of the cultured cortex (Molnár and Blakemore, 1999).

\section{Spontaneous firing in cultured cortex and thalamus}

To investigate whether electrical activity was generated in thalamic and cortical cells in vitro, firing activity was recorded during culturing at several locations of thalamic and cortical explants (Fig. 2A). Spontaneous firing was observed as single units and field potentials in both thalamic and cortical cells (Fig. 2B) (see also Fig. 4A). The single-unit activity lasted a few milliseconds in duration, whereas the field potentials were typified by a negative potential of $5-100 \mathrm{~ms}$ in duration and variable amplitude (Fig. $2 \mathrm{~B}$ ). In most cases, the spontaneous activity appeared rhythmically with a long intervening silent period, which varied among samples. The field potentials tended to be synchronized between thalamus and neocortex after 2 weeks in vitro (Fig. 2C) (see Discussion). The firing activity initiated mostly in the upper layers and propagated to the deep layers and thalamic explants, although propagation took place from the deep layers to the upper layers in some cases (Fig. $2 D$ ). The propagation velocity was $0.2-0.3 \mathrm{~m} / \mathrm{s}$, which was similar to that of stimulus-evoked activity in the coculture preparations $(0.33 \mathrm{~m} / \mathrm{s}$ ) (Yamamoto et al., 1989). Therefore, it is likely that such synchronous activity in cocultured thalamic and cortical explants is attributable to the propagation of action potentials and synaptic transmission rather than a propagating synchronized wave (Feller et al., 1997; Garaschuk et al., 2000).

Development of spontaneous firing was studied by monitoring activity in the same samples for $>1$ week. As shown in Figure $2 E$, spontaneous firing was infrequent before 8 DIV but was prominent at later stages. The quantitative analysis clearly demonstrated that the mean firing rate was quite low in the early stages in both thalamic and cortical explants $(0.58 \pm$ $0.10 \mathrm{~Hz}, n=5$ for $6-9$ DIV in cortical explants; $0.22 \pm 0.06 \mathrm{~Hz}$, $n=5$ for $6-9$ DIV in thalamic explants) but increased strikingly by 10 DIV and persisted at later stages $(2.7 \pm 0.35 \mathrm{~Hz}, n=5$ for $10-14$ DIV in cortical explants; $0.89 \pm 0.28 \mathrm{~Hz}, n=5$ for $10-14$ DIV in thalamic explants) (Fig. $2 F$ ).

\section{The effect of blocking action potential or synaptic transmission on axonal branching}

As described above, both spontaneous activity and axonal branching developed during the second week in vitro. This raises the possibility that spontaneous activity may affect axonal branching. To test this possibility, cocultures were subjected to pharmacological treatment with blockers of sodium channels or glutamatergic synaptic transmission during the second week in vitro. The effects of the pharmacological treatments on cell survival and migration could be minimized by applying these blockers during this period, because these developmental events are almost settled after 1 week in vitro (Götz and Bolz, 1992; Yamamoto et al., 1992, 1997). Indeed, Nissl-stained sections of the treated cultures revealed no obvious differences in either cell size or density from control (data not shown).

As demonstrated in Figure 3A, TC axon branching decreased in these treated cultures after 2 weeks in vitro. Quantitative analysis showed that, in the presence of TTX, APV, or DNQX, the number of branch points fell to one-fifth to one-third of the value in the untreated control group $(3.0 \pm 1.0, n=21, p<0.001$ for TTX; $3.4 \pm 1.2, n=13, p<0.001$ for DNQX; $5.3 \pm 0.86, n=47$, $p<0.001$ for APV) (Fig. 3B). Layer specificity of axonal branch- 
ing was also reduced under these treatments (Fig. 3B). Likewise, application of these blockers drastically decreased the branch length in the laminar locations in which branches were formed abundantly in the control, without a significant change in the deep layers (Fig. 3C).

To investigate what aspects of neural activity may account for the reduction of axonal branching, firing activity was monitored under conditions of chronic drug applications. Addition of TTX to the culture medium quickly caused a complete cessation of spontaneous activity (Fig. 4). This effect persisted to the final day of recording. In the presence of DNQX, spontaneous activity was also transiently diminished but was recovered to some extent $(0.49 \pm 0.09 \mathrm{~Hz}, n=2$ for cortical explants; $0.13 \pm 0.02 \mathrm{~Hz}, n=2$ for thalamic explants) (Fig. 4). Likewise, a transient depression and recovery phase were found under NMDA receptor blockade, although, in the case of APV treatment, the recovery was much more prominent in both thalamic and cortical explants $(1.37 \pm 0.16 \mathrm{~Hz}, n=3$ for cortical explant; $1.45 \pm 0.25 \mathrm{~Hz}, n=3$ for thalamic explant). Thus, TTX treatment thoroughly diminishes firing activity, but blockers of either type of glutamate receptor appear to affect synaptic transmission without permanently reducing firing activity (Uesaka et al., 2005).

\section{The role of neural activity in dynamics of axonal branching}

There are several possible mechanisms for the decrease of axonal branching seen under pharmacological blockade: a decrease in added branches, an increase in retracted branches, a failure of branch stabilization, reduced motility, and so forth. To examine these possibilities, the dynamics of TC axon branching was studied by time-lapse imaging either with or without drug applications. The morphologies of individual axons were observed daily by confocal microscopy.

Figure 5, $A$ and $C$, shows examples of axons imaged for several consecutive days. Over the observation period, TC axon arbors became larger and more complex. The average number of branch points and the total axon length increased gradually as development progressed, but the average branch tip length decreased during culturing (supplemental Fig. 2, available at www.jneurosci. org as supplemental material). Analysis of individual axonal branches revealed that axonal branches did not increase monotonically but remodeled actively (Fig. $5 B$ ). More than $80 \%$ of branches showed changes in length. Branch behaviors were categorized according to the changes in length of individual branches (see Materials and Methods). First, we analyzed the laminar specificity of branch dynamics $(n=12)$. Most added and elongating branches were enriched within $300-500 \mu \mathrm{m}$ from the pial surface (layer 4), although these behaviors were found in the more superficial layers as well (see Fig. 7A). Lost and retracting branches also emerged most frequently within $300-500 \mu \mathrm{m}$, but the distribution was more uniform over all cortical depths.

In drug-treated slices, the total number of branches was sub-
A
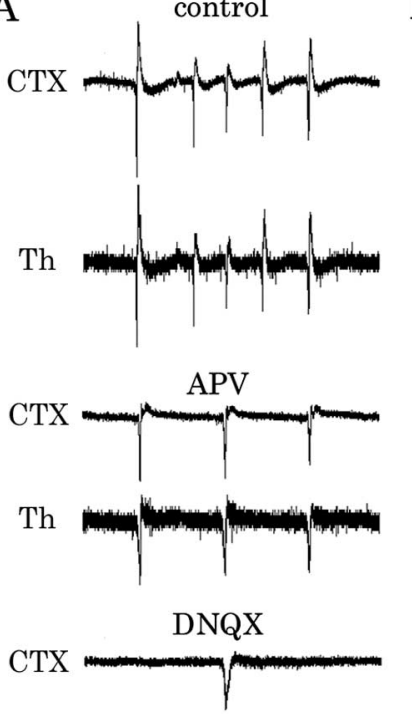

Th

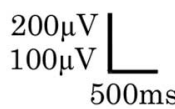

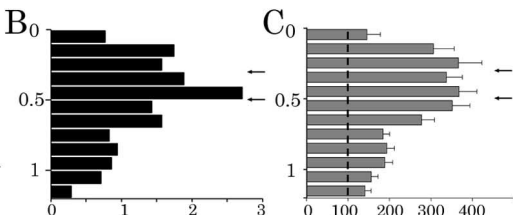
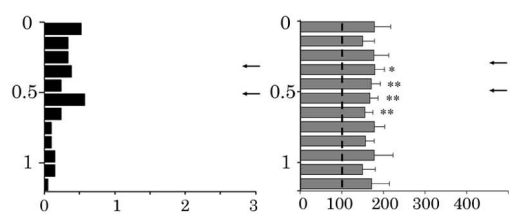
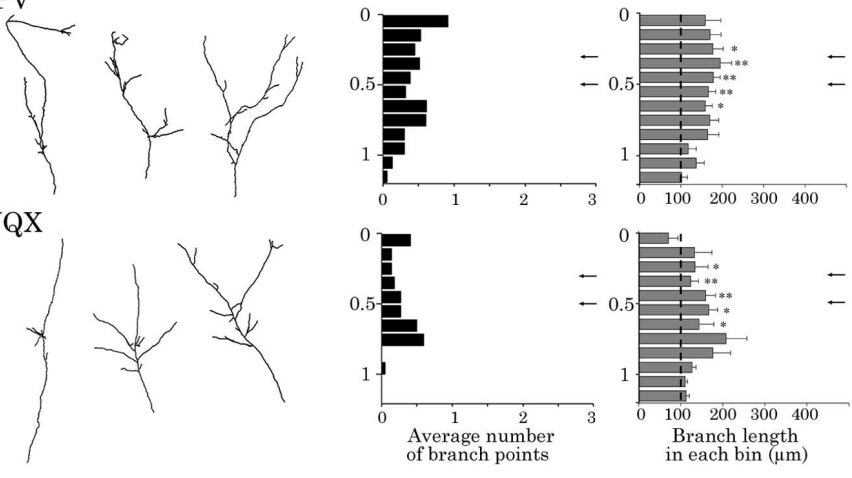

in each bin $(\mu \mathrm{m})$

Figure 3. The effects of blocking spontaneous activity on axonal branching. $A$, Individual TC axons after 2 weeks in vitro in untreated and pharmacologically treated cultures. Typical examples are shown in each treatment. Axons for control are the same 政 列 and treated cultures. Histograms show mean branch length in each bin $(100 \mu \mathrm{m})$ as a function of cortical depth. Interrupted lines represent the minimal length $(100 \mu \mathrm{m})$ in each bin. ${ }^{*} p<0.05$ and ${ }^{* *} p<0.01$, significant differences from the control.
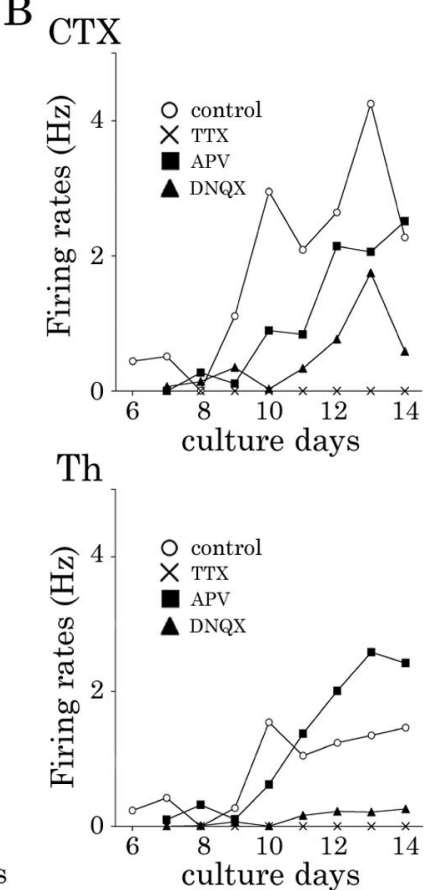

Figure 4. Effects of TTX, DNQX, or APV on spontaneous activity of cortical (CTX) and thalamic (Th) neurons. $\boldsymbol{A}$, The typical patterns of spontaneous activity after 12 DIV under control, DNQX, or APV treatments. $\boldsymbol{B}$, The mean firing rates of spontaneous activity in cortical (top) and thalamic explants (bottom) are shown as a function of culture days. Open circles represent the firing rates for control group ( 5 cultures), filled triangles for DNQX group ( 2 cultures), filled rectangles for APV group ( 3 cultures), and $\times$ for TTX group ( 2 cultures). 

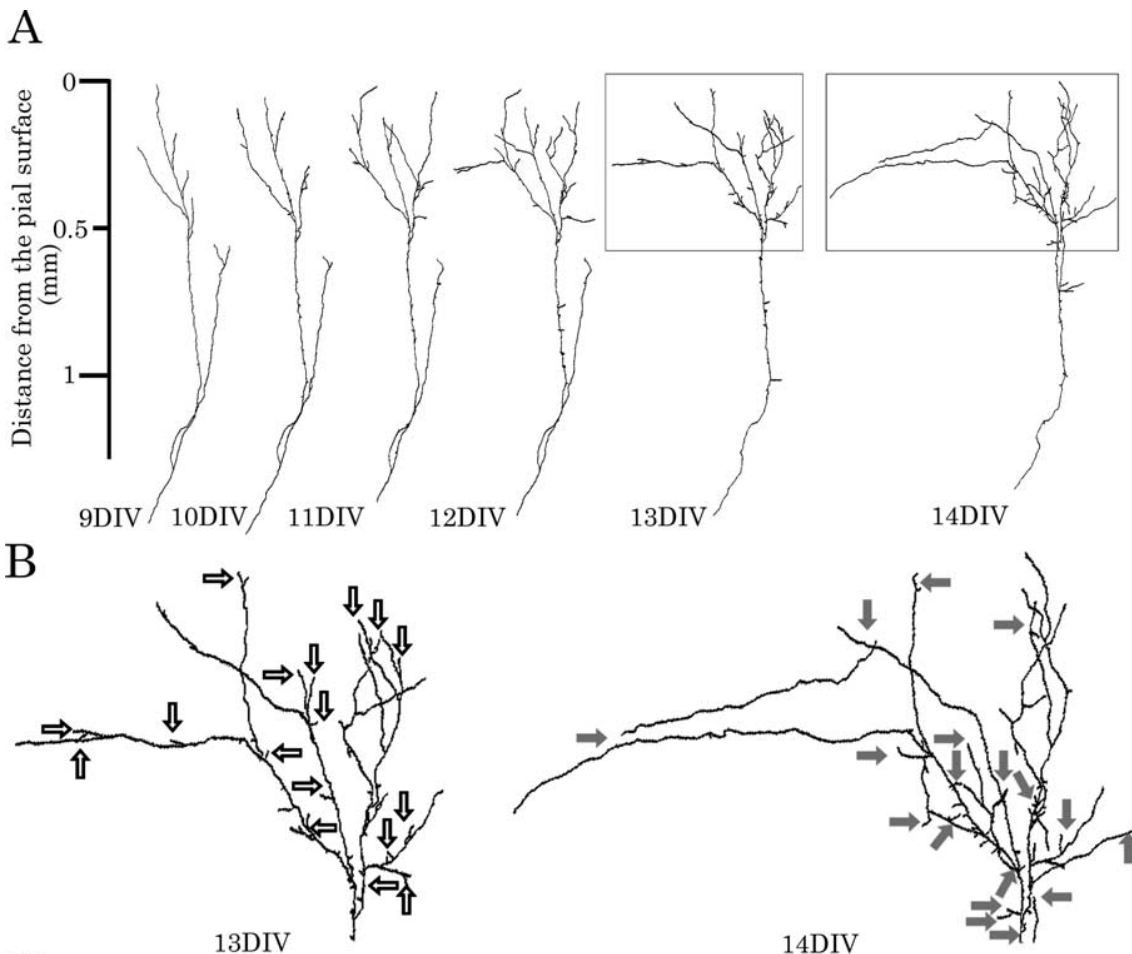

$\mathrm{C}$
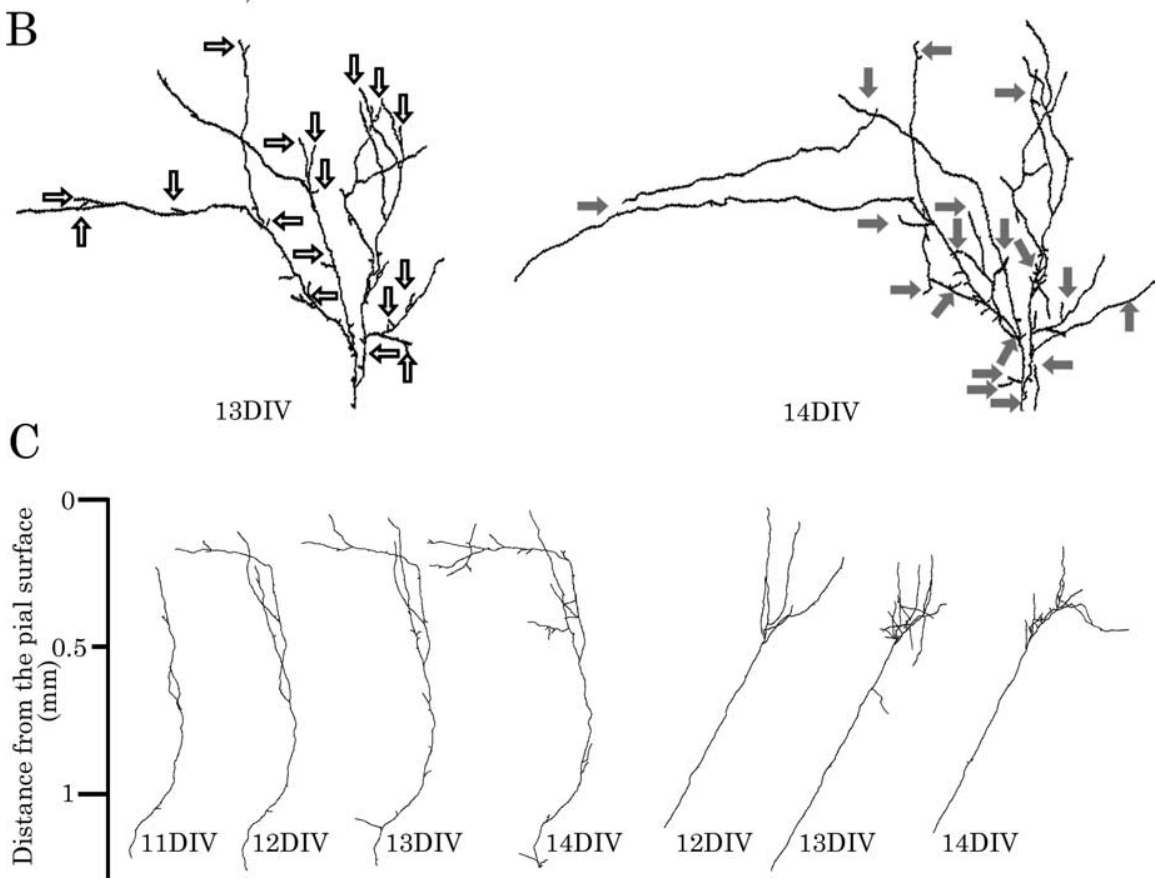

Figure 5. Time-lapse study of TC axon branching. $\boldsymbol{A}$, This axon was followed daily over $6 \mathrm{~d}(9-14 \mathrm{DIV}) . \boldsymbol{B}$, Higher magnification of two boxed regions in $\boldsymbol{A}$ shows examples of added branch (shaded arrows) and lost branch (open arrows). $\boldsymbol{C}$, Another two examples of dynamic axonal arbors.

stantially decreased compared with the untreated group, but $\sim 90 \%$ of the branches were dynamic exhibiting both growth and loss $(n=$ 8 for DNQX and $n=9$ for APV). The laminar distribution of growing and eliminating branches in DNQX-treated slices was nearly constant across all cortical layers (Figs. 6A, 7A). In APV treatment, the laminar distribution was also flat (Figs. $6 B, 7 A$ ).

Because development of axon branching can be attributed to the accumulated difference between the number of added or elongating branches and the number of retracting or lost branches, the differences per day were calculated for the target and nontarget layers. In untreated cultures, the change in the number of branch points per day was $0.82 \pm 0.32$ in layer 4 (300-500 $\mu \mathrm{m}$ from the pia), which was much higher than the value $(0.1 \pm 0.13 ; p<0.05)$ in the deep layers $(600-800 \mu \mathrm{m})$. Thus, the number of branches in the target layer gradually increased as development progressed. In contrast, the difference in layer 4 was dramatically reduced as a result of drug treatment (Fig. $7 B$ ). Conversely, we did not find that stable branches were more prevalent in the target layer under any conditions (Fig. 7C).

Growth motility of branches increased slightly in the presence of these drugs. As shown in Figure $7 D$, the amount of elongation of branches in the control group was $46.1 \pm 3.9 \mu \mathrm{m} / \mathrm{d}(n=$ 12 ), and the amount of retraction observed was $42.3 \pm 3.9 \mu \mathrm{m} / \mathrm{d}(n=12)$. DNQX and APV application tended to increase the growth rate of the added and elongating branches $(67.5 \pm 13.7 \mu \mathrm{m} / \mathrm{d}$, $n=8$ for DNQX; $65.5 \pm 13.6 \mu \mathrm{m} / \mathrm{d}, n=9$ for APV). DNQX treatment also tended to increase the retraction rate of the lost and retracting branches $(58.9 \mu \mathrm{m} / \mathrm{d} ; n=8)$, although the difference was not significant.

\section{Discussion}

The present study demonstrated that the lamina-specific axonal branching of TC axons corresponds to the stages when spontaneous firing activity increases in both thalamic and cortical neurons. The results further showed that TC axon branching patterns are generated dynamically by ongoing branch addition and elimination with a preference toward branch accumulation in the upper layers and that lamina-specific branching with the remodeling process is reduced by blockade of firing or synaptic activity. These findings suggest that neural activity modifies lamina-specific TC axon branching by affecting the remodeling process.

\section{Activity dependency of TC axon branching}

To date, remodeling of branch formation has been shown in several systems. An in vivo imaging study has demonstrated that thalamic axons originating from the medial part of the posterior nucleus of the thalamus form arbors with growth and retraction in layer 1 of the somatosensory cortex (Portera-Cailliau et al., 2005). Similar dynamic axon branching has also been reported in Xenopus retinotectal system (O'Rourke and Fraser, 1990; O’Rourke et al., 1994; Cohen-Cory, 1999; Ruthazer et al., 2003). The present study further demonstrated that such a remodeling process with addition and elimination was predominant in the restricted layers and regulated by neural activity. Indeed, we observed that blocking electrical activity disrupted laminar specificity of axonal branching. This disruption is mediated primarily by a reduction of axonal branch formation in the target layer rather than an expansion of axonal branching in the nontarget layers. One possibility to account for the reduction is that activity blockade merely decreases the overall growth of TC axons (Goldberg et al., 2002). In agreement with this view, the result showed that branch length was reduced in the presence of the blockers. However, the fact that the activity blockade tended to increase the growth rate of individual branches implies that neural activity does not necessarily reduce growth motility. Another explanation is that accumulation of stable branches in the target layer is promoted by neural activity, but this is unlikely because the lamina distribution of stable branches was almost 
constant across all cortical layers in the presence or absence of the blockers. The most plausible mechanism is that neural activity including synaptic transmission enhances branch dynamics with a bias toward more branch addition and elongating branches in the target layer.

In many regions of the developing nervous system, spontaneous calcium and electrical signals have been observed (Feller, 1999). In fact, thalamic and cortical cells generate spontaneous firing activity during development of thalamocortical axon branches (Mirmiran and Corner, 1982; Flint et al., 1997; Weliky and Katz, 1999; Chiu and Weliky, 2001). These findings support the view that spontaneous activity may participate in branch formation in vivo as well. Herrmann and Shatz (1995) showed that TTX infusion in fetal cat brain caused TC axons to bypass layer 4 with little branching. Thus, the proper laminar specificity of the TC axon projection appears to require neural activity (Wilkemeyer and Angelides, 1996; Anderson and Price, 2002). Likewise, axonal branching of intracortical connections is disrupted by the blockade of neural activity (Dantzker and Callaway, 1998; Butler et al., 2001). Moreover, it has been demonstrated that sensory deprivation and blockade of synaptic transmission reduce remodeling of postsynaptic density and dendritic protrusions (Okabe et al., 1999; Lendvai et al., 2000; Zuo et al., 2005; Ruthazer et al., 2006). Remodeling of postsynaptic structures may lead to remodeling of axonal branching.

Some studies have demonstrated that activity blockade causes an expansion of axonal arbors in the retinotectal projection (Reh and Constantine-Paton, 1985; Cohen-Cory, 1999) and produces widespread terminal arborization of retinogeniculate axons in both target and nontarget layers (Sretavan et al., 1988). It has also been suggested that neural activity promotes axonal growth (Goldberg et al., 2002). The different effects on activity blockade between these and the above findings including our present results might be explained by distinct patterns of neural activity under normal conditions or by the different nature of molecular cues for axonal growth and branching in these different systems (Itoh et al., 1997).

It should be emphasized that neural activity leading to synaptic transmission, rather than firing activity, appears to be required for TC axonal branching because chronic glutamate receptor antagonist application did not consistently reduce firing events (Fig. 4) (Uesaka et al., 2005). To date, synchronized activity between presynaptic and postsynaptic cells has been thought to strengthen neural connectivity (Katz and Shatz, 1996). NMDA receptor activity plays a pivotal role in this process as a molecular detector of synchronized activity (Cline and Constantine-Paton, 1990; O’Rourke et al., 1994; Fox et al., 1996; Rajan et al., 1999; Iwasato et al., 2000; Ruthazer et al., 2003; Ohno et al., 2004; Lee et al., 2005). The present findings imply that NMDA receptor activation might also be necessary for TC axon branching, although non-NMDA-type glutamate receptor activation was found to be required for cortical axon branching (Uesaka et al., 2005). The following results support this view. First, synchronized activity between thalamic and cortical cells increased during the second week in vitro, when axonal branching was actively taking place (Fig. 2C) (supplemental Fig. 3A, available at www.jneurosci.org as supplemental material). Second, chronic application of NMDA antagonist reduced axon branching considerably without decreasing firing frequency and synchronous activity (Fig. 4). Third, synchronous activity was reduced by non-NMDA receptor antagonist application, which also reduced branching (supplemental Fig. 3B, available at www.jneurosci.org as supplemental material), that is, the synchronization was lost despite the presence of NMDA receptor. Thus, it is likely that NMDA activation and synchronous activity between thalamic and cortical cells may promote TC axon branching.

It has been demonstrated recently that firing activity of retinal 


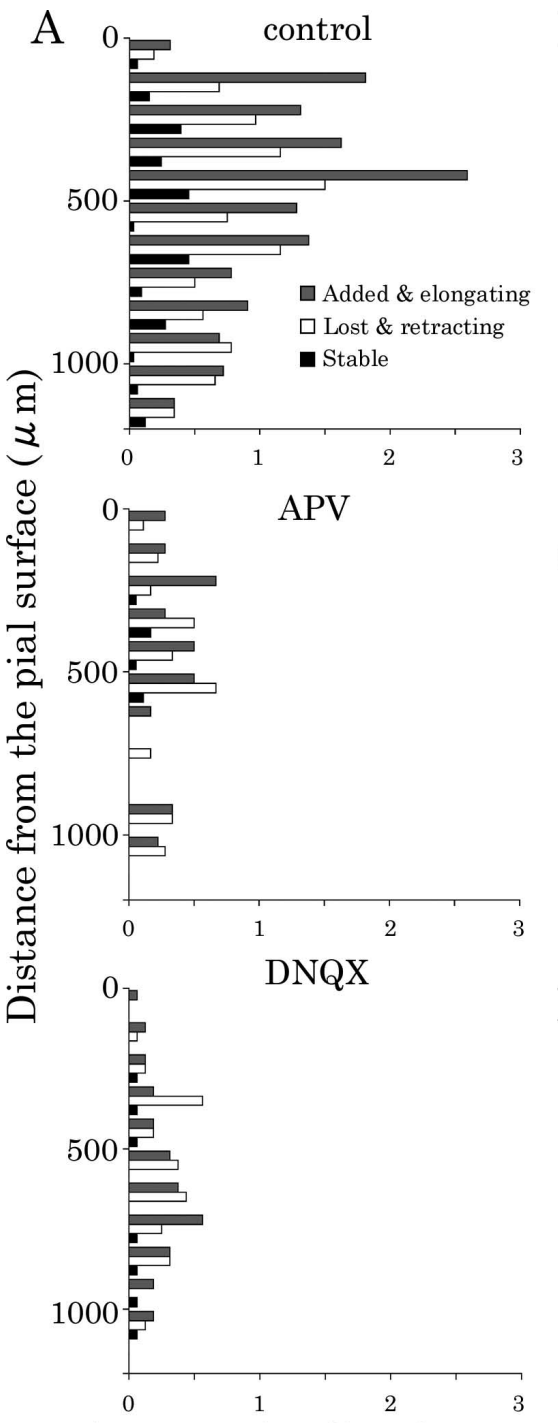

Average number of branching points

Figure 7. Quantification of branch dynamics in the absence or presence of blockers of synaptic activity. $A$, Laminar distribution of growth or retraction of branches in untreated (10 cultures, 12 axons), DNQX-treated ( 6 cultures, 8 axons), and APV-treated (8 cultures, 9 axons) cortical slices. The distributions are plotted against the distance from the pial surface. Gray columns show added and elongating branches. White columns show lost and retracting branches. Black columns show stable branches. $\boldsymbol{B}$, Difference of the number of branch points between growth (added and elongating) and elimination (lost and retracting) branches was evaluated in layer 4 and the deep layers for untreated, DNQX-treated, and APV-treated cultures. Mann-Whitney $U$ test, ${ }^{*} p<0.05$. C, The number of stable branches in layer 4 and deep layers is shown in the absence or presence of each blocker. $\boldsymbol{D}$, Changes in the length of branches. White column shows untreated. Black column shows APV. Gray column shows DNQX. the branch-promoting factor is expressed more abundantly as cortical development proceeds (Molnár and Blakemore, 1999). The present results not only support this view but also suggest that neural activity including synaptic transmission could play a role in regulating the proper expression of these molecules. Alternatively, activity might modify the expression and responsiveness of the receptors on growing TC axons or affect downstream signaling mechanisms, including cytoskeletal changes in TC axons. Although the molecules that underlie this process are essentially unknown, the Eph family of receptor tyrosine kinases and their cell-surfacebound ligands, the ephrins, are plausible candidates. Ephrin-As and EphAs have been implicated to provide information for axon guidance of cortical efferent and afferent, including TC axons (Castellani et al., 1998; Gao et al., 1998; Vanderhaeghen et al., 2000; Yabuta et al., 2000; Mann et al., 2002; Uziel et al., 2002; Dufour et al., 2003; Cang et al., 2005). Moreover, the expression pattern of EphA and the responsiveness to ephrin-As on growing axons has been demonstrated to be regulated by electrical activity (Hanson and Landmesser, 2004; Nicol et al., 2007). N-cadherin and neural cell adhesion molecule are other candidate molecules for TC axon branching, because expressions of these molecules in the developing cortex could be affected by neural activity (Itoh et al., 1997; Schuster et al., 1998; Huntley and Benson, 1999; Bozdagi et al., 2000; Gil et al., 2002).

In conclusion, TC axon branching involves a process of dynamic remodeling that may be regulated by branchpromoting and -inhibiting molecules. Neural activity including synaptic transmission could modulate these molecular mechanisms, indicating an interplay between lamina-specific targeting mechanisms and neural activity.

\section{References}

Agmon A, Yang LT, O'Dowd DK, Jones EG axons, independently of synaptic transmission, is required for a coarse topographic map formation in the rodent retinotectal projection (Nicol et al., 2007). Relative importance of synaptic activity might be different in developmental stages, nervous systems, or guidance properties. Indeed, fine-scale axon remodeling in retinotectal projections has been shown to involve synaptic activity (Cline and Constantine-Paton, 1990; O'Rourke et al., 1994; Rajan et al., 1999; Ruthazer et al., 2003; Hua et al., 2005), which is consistent with our present findings.

\section{Interplay between laminar cues and electrical activity}

The previous study has suggested that branch-promoting molecules are expressed in the target layer, whereas polysialic acid, a branch-inhibiting molecule, is expressed in all cortical layers (Yamamoto et al., 2000). Furthermore, it has been indicated that terminations into layer IV of developing mouse barrel cortex. J Neurosci 13:5365-5382.

Anderson G, Price DJ (2002) Layer-specific thalamocortical innervation in organotypic cultures is prevented by substances that alter neural activity. Eur J Neurosci 16:345-349.

Antonini A, Stryker MP (1996) Plasticity of geniculocortical afferents following brief or prolonged monocular occlusion in the cat. J Comp Neurol 369:64-82.

Antonini A, Fagiolini M, Stryker MP (1999) Anatomical correlates of functional plasticity in mouse visual cortex. J Neurosci 19:4388-4406.

Bolz J, Novak N, Staiger V (1992) Formation of specific afferent connections in organotypic slice cultures from rat visual cortex cocultured with lateral geniculate nucleus. J Neurosci 12:3054-3070.

Bozdagi O, Shan W, Tanaka H, Benson DL, Huntley GW (2000) Increasing numbers of synaptic puncta during late-phase LTP: N-cadherin is synthe-
(1993) Organized growth of thalamocortical axons from the deep tier of 
sized, recruited to synaptic sites, and required for potentiation. Neuron 28:245-259.

Butler AK, Dantzker JL, Shah RB, Callaway EM (2001) Development of visual cortical axons: layer-specific effects of extrinsic influences and activity blockade. J Comp Neurol 430:321-331.

Cang J, Kaneko M, Yamada J, Woods G, Stryker MP, Feldheim DA (2005) Ephrin-as guide the formation of functional maps in the visual cortex. Neuron 48:577-589.

Castellani V, Yue Y, Gao PP, Zhou R, Bolz J (1998) Dual action of a ligand for Eph receptor tyrosine kinases on specific populations of axons during the development of cortical circuits. J Neurosci 18:4663-4672.

Catalano SM, Robertson RT, Killackey HP (1996) Individual axon morphology and thalamocortical topography in developing rat somatosensory cortex. J Comp Neurol 367:36-53.

Chiu C, Weliky M (2001) Spontaneous activity in developing ferret visual cortex in vivo. J Neurosci 21:8906-8914.

Cline H (2003) Sperry and Hebb: oil and vinegar? Trends Neurosci 26:655-661.

Cline HT, Constantine-Paton M (1990) NMDA receptor agonist and antagonists alter retinal ganglion cell arbor structure in the developing frog retinotectal projection. J Neurosci 10:1197-1216.

Cohen-Cory S (1999) BDNF modulates, but does not mediate, activitydependent branching and remodeling of optic axon arbors in vivo. J Neurosci 19:9996-10003.

Dantzker JL, Callaway EM (1998) The development of local, layer-specific visual cortical axons in the absence of extrinsic influences and intrinsic activity. J Neurosci 18:4145-4154.

Dufour A, Seibt J, Passante L, Depaepe V, Ciossek T, Frisen J, Kullander K, Flanagan JG, Polleux F, Vanderhaeghen P (2003) Area specificity and topography of thalamocortical projections are controlled by ephrin/Eph genes. Neuron 39:453-465.

Feller MB (1999) Spontaneous correlated activity in developing neural circuits. Neuron 22:653-656.

Feller MB, Butts DA, Aaron HL, Rokhsar DS, Shatz CJ (1997) Dynamic processes shape spatiotemporal properties of retinal waves. Neuron 19:293-306.

Flint AC, Maisch US, Kriegstein AR (1997) Postnatal development of low $\left[\mathrm{Mg}^{2+}\right]$ oscillations in neocortex. J Neurophysiol 78:1990-1996.

Fox K, Schlaggar BL, Glazewski S, O'Leary DD (1996) Glutamate receptor blockade at cortical synapses disrupts development of thalamocortical and columnar organization in somatosensory cortex. Proc Natl Acad Sci USA 93:5584-5589.

Gao PP, Yue Y, Zhang JH, Cerretti DP, Levitt P, Zhou R (1998) Regulation of thalamic neurite outgrowth by the Eph ligand ephrin-A5: implications in the development of thalamocortical projections. Proc Natl Acad Sci USA 95:5329-5334.

Garaschuk O, Linn J, Eilers J, Konnerth A (2000) Large-scale oscillatory calcium waves in the immature cortex. Nat Neurosci 3:452-459.

Ghosh A, Shatz CJ (1992) Pathfinding and target selection by developing geniculocortical axons. J Neurosci 12:39-55.

Gil OD, Needleman L, Huntley GW (2002) Developmental patterns of cadherin expression and localization in relation to compartmentalized thalamocortical terminations in rat barrel cortex. J Comp Neurol 453:372-388.

Goldberg JL, Espinosa JS, Xu Y, Davidson N, Kovacs GT, Barres BA (2002) Retinal ganglion cells do not extend axons by default: promotion by neurotrophic signaling and electrical activity. Neuron 33:689-702.

Götz M, Bolz J (1992) Formation and preservation of cortical layers in slice cultures. J Neurobiol 23:783-802.

Hanson MG, Landmesser LT (2004) Normal patterns of spontaneous activity are required for correct motor axon guidance and the expression of specific guidance molecules. Neuron 43:687-701.

Herrmann K, Shatz CJ (1995) Blockade of action potential activity alters initial arborization of thalamic axons within cortical layer 4. Proc Natl Acad Sci USA 92:11244-11248.

Hua JY, Smear MC, Baier H, Smith SJ (2005) Regulation of axon growth in vivo by activity-based competition. Nature 434:1022-1026.

Hubel DH, Wiesel TN, LeVay S (1977) Plasticity of ocular dominance columns in monkey striate cortex. Philos Trans R Soc Lond B Biol Sci 278:377-409.

Huntley GW, Benson DL (1999) Neural (N)-cadherin at developing thalamocortical synapses provides an adhesion mechanism for the forma- tion of somatopically organized connections. J Comp Neurol 407:453-471.

Itoh K, Ozaki M, Stevens B, Fields RD (1997) Activity-dependent regulation of $\mathrm{N}$-cadherin in DRG neurons: differential regulation of $\mathrm{N}$-cadherin, NCAM, and L1 by distinct patterns of action potentials. J Neurobiol 33:735-748.

Iwasato T, Datwani A, Wolf AM, Nishiyama H, Taguchi Y, Tonegawa S, Knopfel T, Erzurumlu RS, Itohara S (2000) Cortex-restricted disruption of NMDAR1 impairs neuronal patterns in the barrel cortex. Nature 406:726-731.

Kageyama GH, Robertson RT (1993) Development of geniculocortical projections to visual cortex in rat: evidence early ingrowth and synaptogenesis. J Comp Neurol 335:123-148.

Katz LC, Shatz CJ (1996) Synaptic activity and the construction of cortical circuits. Science 274:1133-1138.

Lee LJ, Iwasato T, Itohara S, Erzurumlu RS (2005) Exuberant thalamocortical axon arborization in cortex-specific NMDAR1 knockout mice. J Comp Neurol 485:280-292.

Lendvai B, Stern EA, Chen B, Svoboda K (2000) Experience-dependent plasticity of dendritic spines in the developing rat barrel cortex in vivo. Nature 404:876-881.

Lund JS, Boothe RG, Lund RD (1977) Development of neurons in the visual cortex (area 17) of the monkey (Macaca nemestrina): a Golgi study from fetal day 127 to postnatal maturity. J Comp Neurol 176:149-188.

Mann F, Peuckert C, Dehner F, Zhou R, Bolz J (2002) Ephrins regulate the formation of terminal axonal arbors during the development of thalamocortical projections. Development 129:3945-3955.

Mirmiran M, Corner M (1982) Neuronal discharge patterns in the occipital cortex of developing rats during active and quiet sleep. Brain Res 255:37-48.

Molnár Z, Blakemore C (1991) Lack of regional specificity for connections formed between thalamus and cortex in coculture. Nature 351:475-477.

Molnár Z, Blakemore C (1999) Development of signals influencing the growth and termination of thalamocortical axons in organotypic culture. Exp Neurol 156:363-393.

Nicol X, Voyatzis S, Muzerelle A, Narboux-Neme N, Sudhof TC, Miles R, Gaspar P (2007) cAMP oscillations and retinal activity are permissive for ephrin signaling during the establishment of the retinotopic map. Nat Neurosci 10:340-347.

Niwa H, Yamamura K, Miyazaki J (1991) Efficient selection for highexpression transfectants with a novel eukaryotic vector. Gene 108:193-199.

Ohno T, Maeda H, Sakurai M (2004) Regionally specific distribution of corticospinal synapses because of activity-dependent synapse elimination in vitro. J Neurosci 24:1377-1384.

Okabe S, Kim HD, Miwa A, Kuriu T, Okado H (1999) Continual remodeling of postsynaptic density and its regulation by synaptic activity. Nat Neurosci 2:804-811.

O'Rourke NA, Fraser SE (1990) Dynamic changes in optic fiber terminal arbors lead to retinotopic map formation: an in vivo confocal microscopic study. Neuron 5:159-171.

O'Rourke NA, Cline HT, Fraser SE (1994) Rapid remodeling of retinal arbors in the tectum with and without blockade of synaptic transmission. Neuron 12:921-934.

Portera-Cailliau C, Weimer RM, De Paola V, Caroni P, Svoboda K (2005) Diverse modes of axon elaboration in the developing neocortex. PLoS Biol 3:e272.

Rajan I, Witte S, Cline HT (1999) NMDA receptor activity stabilizes presynaptic retinotectal axons and postsynaptic optic tectal cell dendrites in vivo. J Neurobiol 38:357-368.

Reh TA, Constantine-Paton M (1985) Eye-specific segregation requires neural activity in three-eyed Rana pipiens. J Neurosci 5:1132-1143.

Ruthazer ES, Akerman CJ, Cline HT (2003) Control of axon branch dynamics by correlated activity in vivo. Science 301:66-70.

Ruthazer ES, Li J, Cline HT (2006) Stabilization of axon branch dynamics by synaptic maturation. J Neurosci 26:3594-3603.

Schuster T, Krug M, Hassan H, Schachner M (1998) Increase in proportion of hippocampal spine synapses expressing neural cell adhesion molecule NCAM180 following long-term potentiation. J Neurobiol 37:359-372.

Shimono K, Baudry M, Panchenko V, Taketani M (2002) Chronic multichannel recordings from organotypic hippocampal slice cultures: protection from excitotoxic effects of NMDA by non-competitive NMDA antagonists. J Neurosci Methods 120:193-202. 
Sretavan DW, Shatz CJ, Stryker MP (1988) Modification of retinal ganglion cell axon morphology by prenatal infusion of tetrodotoxin. Nature 336:468-471.

Uesaka N, Hirai S, Maruyama T, Ruthazer ES, Yamamoto N (2005) Activity dependence of cortical axon branch formation: a morphological and electrophysiological study using organotypic slice cultures. J Neurosci 25:1-9.

Uziel D, Muhlfriedel S, Zarbalis K, Wurst W, Levitt P, Bolz J (2002) Miswiring of limbic thalamocortical projections in the absence of ephrin-A5. J Neurosci 22:9352-9357.

Vanderhaeghen P, Lu Q, Prakash N, Frisen J, Walsh CA, Frostig RD, Flanagan JG (2000) A mapping label required for normal scale of body representation in the cortex. Nat Neurosci 3:358-365.

Weliky M, Katz LC (1999) Correlational structure of spontaneous neuronal activity in the developing lateral geniculate nucleus in vivo. Science 285:599-604.

Wilkemeyer MF, Angelides KJ (1996) Addition of tetrodotoxin alters the morphology of thalamocortical axons in organotypic cocultures. J Neurosci Res 43:707-718.
Yabuta NH, Butler AK, Callaway EM (2000) Laminar specificity of local circuits in barrel cortex of ephrin-A5 knock-out mice. J Neurosci 20:RC88(1-4).

Yamamoto N, Kurotani T, Toyama K (1989) Neural connections between the lateral geniculate nucleus and visual cortex in vitro. Science 245:192-194.

Yamamoto N, Yamada K, Kurotani T, Toyama K (1992) Laminar specificity of extrinsic cortical connections studied in coculture preparations. Neuron 9:217-228.

Yamamoto N, Higashi S, Toyama K (1997) Stop and branch behaviors of geniculocortical axons: a time-lapse study in organotypic cocultures. J Neurosci 17:3653-3663.

Yamamoto N, Inui K, Matsuyama Y, Harada A, Hanamura K, Murakami F, Ruthazer ES, Rutishauser U, Seki T (2000) Inhibitory mechanism by polysialic acid for lamina-specific branch formation of thalamocortical axons. J Neurosci 20:9145-9151.

Zuo Y, Lin A, Chang P, Gan WB (2005) Development of long-term dendritic spine stability in diverse regions of cerebral cortex. Neuron 46:181189. 